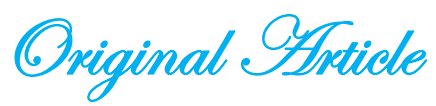

\title{
The use of height versus weight in determining praziquantel dose for treatment of Schistosomiasis in children Mohammed Osman Swar ${ }^{1 *}$ and Mutamad Amin ${ }^{1}$
}

\begin{abstract}
Background: Praziquantel (PZQ) is currently the drug of choice in treatment of Schistosomiasis because of its high efficacy, few and transient side effects, simple administration and competitive cost. In community-based management and during mass chemotherapy of Schistosomiasis, WHO recommended a dose pole where height is taken as a single parameter to select the required dose.

Objective: To compare PZQ dose according to WHO dose pole with the calculated weightdependent dose and test their consistency.

Method: We used a growth percentile ruler (MOSWAR) that includes weight, height, head circumference and body surface area according to age and sex. Data were then compiled into a table-form that included PZQ dose according to the dose pole and the calculated mean weightdependent dose.

Results: The results showed that PZQ dose according to the dose pole is not consistent with the weight-dependent dose.

Conclusion: During mass chemotherapy and community-based management of Schistosomiasis in children, we recommend the use of a similar table or a device similar to (MOSWAR) to determine PZQ dose according to whichever of the growth parameters is available.
\end{abstract}

Keywords: PZQ, Dose pole, infantometer, stadiometer.

$\mathrm{T}$ he World Health Organization (WHO) estimated that 200 million people are infected with Schistosomiasis and 600 million are at risk of infection in 76 countries. Recently, at risk population has been updated to 779 million ${ }^{1}$. Many of those with Schistosomiasis are in the Sub-Saharan Africa. It is estimated that 14 million preschool children in this region are infected with s. haematobium and 4.6 million with s. mansoni ${ }^{2}$. In Ghana, a parasitological prevalence of $11.2 \%$ for $\mathrm{s}$. haematobium in infants of four months is now documented ${ }^{3}$, while in Uganda, it is reported that nearly $50 \%$ of children under- three years of age living along the northern shoreline of Lake Victoria had s .mansoni infection ${ }^{4}$. In Niger, infection rates of $s$. haematobium in young children and their mothers are reported to be $60.5 \%$ and $72.2 \%$ respectively $^{5}$. Taking into consideration that in community-based management of Schistosomiasis and

\footnotetext{
1. Ahfad University for Women

* Correspondence: M.O.Swar, Head, Paediatrics and Child Health Unit, SOM, AUW

E-mail:moswar@hotmail.com
}

during mass treatment campaigns, it is not always possible to have durable and efficacious weighing scales to apply weightdependent doses, WHO recommended a dose pole for Praziquantel (PZQ), as a primary agent in treatment of Schistosomiasis, that uses height as a single parameter to calculate the dose of PZQ with the lowest height division of $94 \mathrm{~cm}$ for a dose of one tablet $(600$ $\mathrm{mg}$ ) and a highest division of $\geq 178 \mathrm{~cm}$ for a dose of five tablets ${ }^{6}$. This dose pole did not consider children with growth faltering, short stature due to high prevalence of malnutrition or other debilitating chronic diseases and tall stature secondary to ethnic variations within the same region. Furthermore, it was observed that there is a treatment gap in the use of PZQ for treatment of Schistosomiasis since most of the previous studies included children more than six years old and adults ${ }^{7}$. This gap included children younger than 6 years who are indirectly exposed to infection by using contaminated water for bathing or washing cloths of infected children in pools and canals. Consequently, in recent studies done 
in Uganda, the dose pole was amended to accommodate for younger age groups i.e. less than five years old and it was suggested that WHO lower limit for a dose of one tablet is to be adjusted to $>99 \mathrm{~cm}-110 \mathrm{~cm}$ instead of the previous one of $94 \mathrm{~cm}$. The extended dose pole added two new height intervals: $60-$ $84 \mathrm{~cm}$ for one half of a tablet and $>84-99 \mathrm{~cm}$ for three quarters of a tablet ${ }^{8}$.

Our study is planned to compare PZQ heightdependent dose according to the WHO extended pole $(60-178 \mathrm{~cm})$ with the weight dependent dose $(40 \mathrm{mg} / \mathrm{kg}$ body weight) and assess their consistency.

\section{Methodology}

For exploring the validity of PZQ dose by comparing the extended WHO dose pole (60 - $178 \mathrm{~cm})$ and weight-dependent dose ( $40 \mathrm{mg} / \mathrm{kg}$ body weight), we used a handy, durable and easy to use growth percentile ruler that includes World Health Organization/National Center for Health Statistics -U.S.A records of weight and height according to age and sex. The ruler (MOSWAR) contains age for children from birth to 18 years and gender on two different faces (Fig.1).

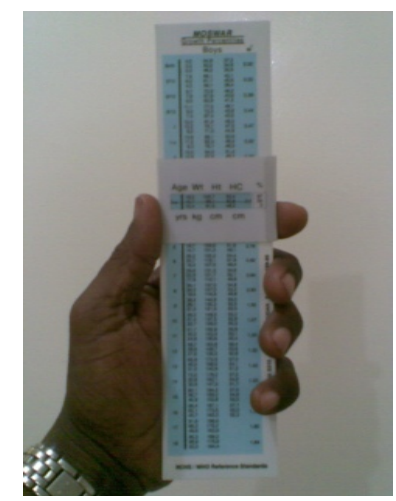

Fig.1: MOSWAR growth percentile ruler (pink: girls, blue: boys)

A sliding transparent window, when moved up and down the ruler, locates weight, height, head circumference and body surface area for the selected age with the $3^{\text {rd }}, 50^{\text {th }}$ and $97^{\text {th }}$ percentiles apparent along three horizontal lines within the transparent window. Weight of children is determined by using the data shown on (MOSWAR) according to the corresponding height selected from the dose pole. All data were then transformed into a table form (Table 1) that included WHO dose pole and the corresponding age, weight and height as seen on (MOSWAR). Heightdependent dose according to WHO dose pole, the calculated mean weight-dependent dose (Mean $=\sum \div \mathrm{n}$ ) and the calculated difference between the height-dependent dose and the mean weight-dependent dose are shown in different columns.

\section{Results}

Our results showed that PZQ dose when given according to the dose pole at the age group $<5$ years is less than the weightdependent dose. At age group $>5-8$ years and age group $>12-18$ years it is almost equal to the weight-dependent dose while at age group $8-12$ years and age $\geq 18$ years it is more than the weight -dependent dose.

\section{Discussion}

The results of this study shows that PZQ dose when given according to the $\mathrm{WHO}$ dose pole is not consistent with the dose when calculated according to body weight. These results are compatible with the reports from Uganda where they noted that height is not a very adequate tool to determine the standard PZQ dose and that there was a significant difference in each dosing category when height instead of weight is used as a dosing tool, possibly due to the high prevalence of malnutrition that leads to growth retardation. Gender and ethnic variations should also be considered in this context. In addition, it is always difficult to assess length and height in children $<5$ years without having proper scales i.e. Infantometer or stadiometer respectively and well trained staff. At age $\geq 18$ years, growth velocity decelerates and the body surface area is affected by weight rather than height changes since it is the muscle mass and body fat that changes at this age group rather than height, hence height alone might not be a dependable parameter to estimate PZQ dose. Dose preparation and administration, using the currently available tablets of $600 \mathrm{mg}$ is rather inaccurate and cumbersome. 
Table 1: Praziquantel dose/ height (WHO dose pole) Compared to dose/ weight (40mg/kg)

\begin{tabular}{|c|c|c|c|c|c|c|c|}
\hline Dose Pole $(\mathrm{cm})$ & Dose/Ht (mg) & Corresponding Age & $\begin{array}{r}\text { Corresponding } \\
\text { Weight }(\mathrm{kg})\end{array}$ & Dose/Wt (mg) & $\begin{array}{l}\text { Mean Dose / } \\
\text { Wt (mg) }\end{array}$ & $\begin{array}{l}\text { Difference between } \\
\text { Dose/Ht and Dose } \\
\text { /Wt (mg) }\end{array}$ & $\begin{array}{l}\text { Dose/Ht } \\
\text { compared to } \\
\text { Dose/Wt }\end{array}$ \\
\hline $60-84$ & $1 / 2 \operatorname{tab}(300)$ & 3 months $-1 \frac{1}{2} \mathrm{yr}$ & $5.6-11.5$ & $224-460$ & 342 & 42 & $\downarrow$ \\
\hline$>99-110$ & 1 tab (600) & $>3 \frac{1}{2} \mathrm{yr}-5 \mathrm{yr}$ & $>15.3-18.4$ & $612-736$ & 674 & 74 & $\downarrow$ \\
\hline$>110-124$ & $1 \frac{1}{2}$ tabs $(900)$ & $>5 \mathrm{yr}-8 \mathrm{yr}$ & $>18.4-25.3$ & $736-1012$ & 874 & 26 & $\approx=$ \\
\hline $125-137$ & 2 tabs $(1200)$ & $>8 \mathrm{yr}-10 \mathrm{yr}$ & $>25.3-31.4$ & $1012-1256$ & 1134 & 66 & $\uparrow$ \\
\hline $138-149$ & $2 \frac{1}{2}$ tabs $(1500)$ & $>10 \mathrm{yr}-12 \mathrm{yr}$ & $>31.4-39.8$ & $1256-1592$ & 1428 & 72 & $\uparrow$ \\
\hline $160-177$ & 4 tabs $(2400)$ & $>14 \mathrm{yr}-18 \mathrm{yr}$ & $>50.8-68.9$ & $2032-2756$ & 2394 & 4 & $\approx=$ \\
\hline$\geq 178$ & 5 tabs $(3000)$ & $>18 \mathrm{yr}$ & $\geq 69$ & 2780 & & 210 & $\uparrow$ \\
\hline
\end{tabular}


The required dose should be prepared by breaking the tablets into small pieces or by crushing it and adding to it a honey-based solution or a sweet drink to make palatable for children to drink. Furthermore, it is impractical to use weight alone as a single parameter to calculate PZQ dose since weighing scales are not always available in peripheral areas and they need regular maintenance and calibration. The table shown above and the growth percentile ruler that includes weight and height of children at different ages and sex are practical tools that can be used in health settings and mass chemotherapy campaigns until an efficient and cost-effective PZQ syrup formulation with long shelf-life is produced or an age dependent dosing system is approved.

\section{Conclusion}

PZQ is a safe, efficient and cost-effective drug when used in treatment of Schistosomiasis during mass chemotherapy campaigns or in treatment of isolated patients. Data derived from this study indicate that PZQ dose when used according to the suggested dose pole is not consistent with the dose calculated according to weight. We recommend a chart similar to the table that we used or a handy ruler that includes weight and height of children at different ages and sex to be used in community research work and during mass treatment campaigns to determine PZQ weight-dependent dose according to whichever growth parameter is available.

\section{References}

1. Amin M.A, Elhussain D. Challenges in prevention and control of Schistosomiasis in the Sudan. Sudan Journal of Medical Sciences 2009; 4: 79-82.

2. Van der Worf M J, de Vlas S J, Brooke S et al. Quantification of clinical morbidity associated with schistosoma infection in sub-Saharan Africa. Acta Tropica 2003;86: 125-139

3. Bosompem K M, Bentum IA, Otchere J et al. Infant Schistosomiasis in Ghana: a survey in an irrigation Community. Trop Med Int Health 2004;9:917-922

4. Odogwu S, Ramamurthy NK, Kabatereine NB et al. Schistosoma mansoni in infants (aged less than 3years) along Uganda shorelines of Lake Victoria. Ann.Trop Med Parasitol2006; 100:315-326

5. Garba A, Pion S, Cournil A et al. Risk factors for Schistosoma haematobium infection and morbidity in two villages with different transmission. Acta Tropica 2010;115(1-2):84-89

6. World Health Organization (2006): Preventive Chemotherapy in Human Helminthiasis

7. Stothard \& Gabrielli. Schistosomiasis in African infants and preschool children: to treat or not to treat. Trends Parasitol 2007; 23:83-86

8. Figueiredo J C, Pleasant J, Day M et al. Treatment of Intestinal Schistosomiasis in Ugandan pre-school children: best diagnosis, treatment efficacy and side effects, and an extended Praziquantel dosing pole. Int. Health 2010;2:103-113. 